\title{
L'enjeu des aménagements forestiers au Sénégal
}

Entre le hasard écologique et la nécessité politique

\section{Laurence Boutinot}

\section{CpenEdition}

Journals

Édition électronique

URL : http://journals.openedition.org/anthropodev/477

DOI : 10.4000/anthropodev.477

ISSN : 2553-1719

Éditeur

APAD - Association pour l'anthropologie du changement social et du développement

Édition imprimée

Date de publication : 1 avril 2014

Pagination : 157-184

ISBN : 979-10-93476-00-1

ISSN : 2276-2019

\section{Référence électronique}

Laurence Boutinot, «L'enjeu des aménagements forestiers au Sénégal », Anthropologie \&

développement [En ligne], 37-38-39 | 2014, mis en ligne le 01 décembre 2016, consulté le 19 avril 2019.

URL : http://journals.openedition.org/anthropodev/477 ; DOI : 10.4000/anthropodev.477

La revue Anthropologie \& développement est mise à disposition selon les termes de la Licence Creative Commons Attribution 4.0 International. 


\title{
L'enjeu des aménagements forestiers au Sénégal
}

\section{Entre le hasard écologique et la nécessité politique}

\author{
Laurence Boutinot
}

Cet article traite de la particularité du système de production forestier de charbon de bois au Sénégal qui s'illustre dans l'oligopole que forment les grands exploitants urbains, dakarois, traditionnels. Depuis le début des années 2000, ces derniers s'accommodent fort mal des lois sur la décentralisation promulguées en 1996 qui autorisent désormais les conseils ruraux à contrôler l'accès aux forêts situées sur leur territoire administratif. Des aménagements forestiers ont été réalisés dans le cadre d'un projet financé par la Banque Mondiale. II permettait aux communes rurales de capter une part des redevances forestières et aux villageois de devenir eux-mêmes producteurs de charbon de bois, en concurrence avec les grands exploitants. Pour maintenir leur contrôle sur l'accès aux ressources, ces derniers ont institué, avec les services forestiers traditionnels, des zones de production contrôlée (ZPC) dans lesquelles un aménagement sommaire de la ressource les autorisait à bénéficier du même allègement fiscal que celui des zones aménagées. Le ménagement politique des grands patrons charbonniers d'un côté, l'ouverture démocratique du marché de charbon de bois de l'autre, pourraient bien augurer des changements dans les rapports de pouvoir au sein de la filière, dans laquelle l'argumentaire écologique a finalement peu de poids.

This article discusses the particular system forest charcoal production in Senegal which illustrated in the oligopoly formed by the big traditional urban farmers. Since the early 2000s, they accommodate themselves badly of decentralization laws enacted in 1996 that now allow rural councils to control access to forests within their adminis-

${ }^{1}$ Cet article est une version reprise en grande partie et actualisée de Boutinot, L. et C.N., Diouf, 2007, Les linéaments de la politique forestière dans les normes de régulation institutionnelle de la filière du bois énergie au Sénégal, Afrique Contemporaine, 222/2, pp. 57-82, avec l'accord du co-auteur. 
trative territory. Some forest management was carried out under a project funded by the World Bank. It allowed rural communities to capture a share of royalties and forest villagers themselves become producers of charcoal, compete with urban farmers. To maintain their control over access to forest resources, they have established, with traditional forest services, areas controlled output, in which a summary management plan of resource allowed them to enjoy the same tax relief as managed areas. Political gently big charcoal bosses on one side, the democratic opening of the market of charcoal on the other, could initiate changes in power relations within the sector, in which the ecological argument has finally little weight.

\section{Introduction}

La particularité du système de production forestier de bois énergie et de charbon de bois au Sénégal s'illustre dans l'oligopole que forment les grands exploitants urbains, dakarois, traditionnels. Depuis le début des années 2000, ces derniers s'accommodent fort mal des lois sur la décentralisation promulguées en 1996. En transférant la compétence de la gestion des ressources naturelles aux collectivités locales, ces lois autorisent désormais les conseils ruraux à contrôler l'accès aux forêts situées sur leur territoire administratif. $\mathrm{Si}$, jusque là, les grands exploitants ont pu maintenir leur pouvoir sur la filière de production de bois énergie (charbon de bois), c'est sans doute parce que la politique de quotas de production leur permettait de gagner le marché dès lors qu'ils pouvaient se prévaloir d'un capital d'investissement et d'une main d'œuvre disponible. Le quota est une quantité de stères de bois ou de charbon de bois autorisée à la vente quels que soient les lieux de coupe, les essences concernées et les superficies d'exploitation nécessaires pour les obtenir (Ribot, 1990).

La décentralisation vient contredire cette politique car il ne s'agit plus d'exploiter n'importe quel espace forestier pour atteindre une quantité donnée de charbon de bois. D'un point de vue écologique, la production de charbon de bois est désormais liée à la capacité de régénération d'un potentiel forestier qui aura été estimé auparavant dans un espace identifié et délimité. D'autre part, le transfert d'autorité sur la gestion des ressources forestières vers les conseils ruraux autorise théoriquement ces derniers à accepter ou non l'exploitation du bois sur leur territoire, à parti- 
ciper à la délimitation des espaces de production et à négocier la répartition des taxes et redevances. Ce changement n'est toutefois pas sans conditions. Les textes sur la décentralisation et le code forestier de 1998 rendent obligatoire la mise en place, par les conseils ruraux, d'un plan de gestion pour les forêts situées dans leur circonscription. En vérité, ce sont des plans d'aménagement précis qui sont requis pour les espaces forestiers quand ceux-ci sont destinés à la production de charbon de bois. Ces plans nécessitent les compétences techniques et scientifiques que détiennent les agents des services forestiers, que ceux-ci soient détachés dans des projets de développement financés par des bailleurs de fonds ou qu'ils soient restés dans le service public traditionnel des Eaux et Forêts.

La gestion d'une forêt communautaire devient un enjeu de taille où l'on voit s'affronter des acteurs anciens et nouveaux. En cela, elle est un champ de recherche où " il faut chaque fois rechercher les formes spécifiques entre le nouvel entrant qui essaie de faire sauter les verrous du droit d'entrée et le dominant qui essaie de défendre le monopole et d'exclure la concurrence " (Bourdieu, [1984] 2002 :113). Une difficulté vient s'ajouter à l'analyse car, dans le contexte des changements politiques liés à la démocratisation et à la décentralisation qu'on peut observer un peu partout en Afrique de l'Ouest, les formes nouvelles de gouvernance locale brouillent les frontières entre les services publics de l'Etat, les organisations privées et les projets de développement, entre les échelles nationale et locale (Olivier de Sardan et Tidjani Alou, 2009 : 3).

L'aménagement d'une forêt consiste en la délimitation d'un espace, luimême subdivisé en un certain nombre de parcelles qui seront exploitées selon une rotation annuelle. Ainsi, un découpage en huit parcelles distinctes permet à la première parcelle coupée de se régénérer durant les 8 années suivantes avant d'être de nouveau coupée. L'intérêt de l'aménagement d'une forêt réside à la fois dans le souci de maintenir la durabilité de la ressource et dans celui d'attirer les exploitants vers ces zones grâce à un allègement des taxes sur la production qui en est issue. Mais ce programme d'aménagement a également pour but d'inviter les acteurs villageois et les conseils ruraux à participer à la gestion de leur forêt et, partant, à bénéficier des retombées de la production, voire à produire eux-mêmes. Notre article n'aborde pas l'analyse de l'efficacité écologique des aménagements qu'ils soient ou non d'une réelle complexité technique, pas plus qu'il ne se prononce sur leur bien fondé. Nous nous 
attachons plus particulièrement aux réformes du système de gestion des forêts dans leur processus de mise en œuvre, c'est-à-dire " aux actions en train de se faire ", dans une approche des politiques publiques qui rend compte des interactions entre la base et les décideurs et qui rompt avec I'idée d'une faible pénétration des préoccupations nationales au niveau local (Duran, 2004).

Dans un premier temps, cet article reprend la logique des démarches des aménagements forestiers principalement mis en œuvre à partir de 2004 dans le cadre des politiques des énergies domestiques de la Banque Mondiale et du PROGEDE ${ }^{2}$. Cette étude s'appuie sur nos investigations qui se sont étendues entre 2002 et 2007 et qui se sont basées sur des observations participantes notamment lors des réunions relatives à la mise en place des contrats de gestion dans le cadre de l'aménagement de forêts communautaires par le PROGEDE, dans la région de Kolda mais aussi de Tambacounda, lors de l'évaluation de ce projet par une délégation de la Banque Mondiale et lors des séances de délibérations des conseils ruraux. Elle a aussi été le lieu d'entretiens réalisés tant auprès des villageois, charbonniers ou non, des surghas ${ }^{3}$, des patrons charbonniers, des représentants des comités villageois de gestion et des présidents et membres des conseils ruraux et régionaux, qu'auprès des agents forestiers et des représentants du PROGEDE, de la Direction des Eaux et Forêts et des bailleurs de fonds ${ }^{4}$. Elle puise également ses données dans un grand nombre de rapports rédigés par les animateurs de terrain jusqu'aux concepteurs du projet, ainsi que dans les textes de lois, et sur une mise à jour documentaire jusqu'en 2011 (rapports d'évaluation de projet, Journaux officiels, presse). Elle s'appuie ainsi sur une proximité prolongée, aussi bien dans les villages

\footnotetext{
2 Programme de Gestion Durable et Participative des Energies Traditionnelles et de Substitution (PROGEDE). II s'agit d'un projet de développement financé par la Banque Mondiale, le Fonds Mondial pour l'Environnement, les Pays-Bas et une participation du gouvernement sénégalais. Projet déjà évoqué dans l'article Antona M., Boutinot L. et Kassibo B., dans le même numéro.

${ }^{3}$ Les surghas sont des ouvriers charbonniers qui travaillent en forêt pour le compte d'un entrepreneur et sous la surveillance d'un contreplace (ou contremaître).

${ }^{4}$ Une partie de cette étude a contribué à l'évaluation de la participation villageoise à l'aménagement forestier et de l'intégration du PROGEDE à la politique de décentralisation (voir Boutinot, 2004).
} 
riverains, et plus spécifiquement dans ceux de l'une des forêts aménagées par le PROGEDE, qu'auprès des services forestiers. Ces données rendent compte des rapports sociaux observés entre les acteurs : agents forestiers, grands exploitants, villageois mis au travail pour la gestion et, pour certains, producteurs eux-mêmes de charbon de bois, représentants des comités ad hoc pour gérer les forêts et élus locaux.

Dans un deuxième temps et à partir de la description de cette dynamique sociale, l'article poursuit l'analyse sur l'émergence de ce qu'on appelle les «zones de production contrôlée » (ZPC). Ces dernières apparaissent au moment où il devient évident pour les grands exploitants dakarois que les zones aménagées pour la production de charbon de bois sont autant d'espaces qui sont chaque jour un peu plus soustraits à leur contrôle. Ce statut de ZPC est créé par les services forestiers en collaboration avec les grands exploitants. Inspirée des marchés ruraux mis en place au Niger et au Mali voisins ${ }^{5}$, cette troisième voie située entre les zones aménagées (ZA) et les zones non aménagées (ZNA), permet d'alléger les dispositifs de mise en aménagement (inventaire simplifié de la ressource, délimitation et marquage des arbres sommaires, contrôle aléatoire) tout en bénéficiant du même allègement fiscal. Elle se justifie également par une simplification des contrats locaux de gestion avec les communautés rurales. Affichant néanmoins un souci écologique, les agents forestiers du service traditionnel s'autorisent de cette manière à maîtriser, à leur tour, une part de la production de charbon dans des espaces hors du PROGEDE. Tandis que les grands exploitants dakarois, "disposant de redoutables moyens de pression sur les pouvoirs publics » (Ngom, 1996 : 9-10; Ribot, 1990), y ont vu le moyen de garder la mainmise sur les zones de production, passant outre les contraintes d'accès aux zones aménagées et les réformes de la décentralisation.

Nous conclurons sur la question de la pérennité de ces ZPC depuis le retour du PROGEDE phase $2^{6}$ et le changement de gouvernement en 2012. Stratégies de la part des services forestiers et des patrons charbonniers pour contourner les dispositifs d'aménagement participatifs et décentralisés du PROGEDE et pour maintenir leur pouvoir sur le marché de charbon

${ }^{5}$ Cf. Article de Antona M., et al. op.cit.

${ }^{6}$ La phase 1 s'est déroulée de 1998 à 2008. 
de bois, les ZPC posent question. Cependant, l'incertitude qui entourait le financement de la seconde phase du PROGEDE n'était pas sans lien et a permis que ces espaces intermédiaires de production gagnent en reconnaissance et en nombre. Quand bien même elles ne seraient qu'une étape transitoire dans les réformes forestières de ces dernières années, ces ZPC soulèvent les paradoxes de la "libéralisation » de la filière de charbon de bois, souhaitée par les bailleurs de fonds extérieurs.

\section{Les instruments des politiques de gestion forestière}

Le Sénégal se distingue de ses voisins pour le moins sur deux aspects essentiels. D'une part, un taux d'urbanisation fort important et en croissance rapide. Estimée à $25 \%$ de la population en 1987 (Ribot, 1990 : 140), la population urbaine est évaluée en 1998 à $41 \%$ et atteint désormais $47,7 \%$ de la population totale (soit 6105448 habitants sur 12855 153) en 2011 (ANSD, 2011). D'autre part, une préférence de la part des ménages urbains, de l'ordre de $80 \%$ d'entre eux, pour la consommation de charbon de bois, tandis que les zones rurales sénégalaises, de même que des pays comme le Niger et le Mali, consomment davantage de combustibles sous la forme de bois mort.

Les différences du Sénégal tiennent également au fait de l'âge séculaire des processus de décentralisation administrative et politique (Boutinot, 2002). Depuis 1996, dernière réforme en date, les collectivités locales (communes rurales) sont dotées d'organes autonomes pourvus de 10 domaines de compétences légales transférées, dont celui de la gestion du foncier et des ressources naturelles, et d'un conseil élu au suffrage universel. Au niveau de ses ressources, le Sénégal était considéré comme un pays boisé jusque dans les années 1950, bien que comprenant surtout des savanes arbustives et arborées, excepté en Casamance. Les préoccupations environnementales relatives à l'exploitation forestière pour les combustibles ligneux ont peu à peu grandi face au constat des superficies déboisées annuellement. En 1978, le couvert forestier au Sénégal était estimé à $71 \%$ de la superficie totale du pays (Minvielle, 1999 : 113). Ce taux diminuerait environ de 1,2 \% par an (Banque Mondiale 1983 : 22, cité par Ri- 
bot, $1990: 145)$, ce qui représenterait une déforestation annuelle approximative de 165000 hectares en $1990^{7}$ (Ribot, 1990). Or, les chiffres de la FAO à la même période (1992) font état d'une déforestation d'environ 80000 ha par an. Les difficultés à définir les contours d'une forêt restent visibles à travers ces estimations diverses. Selon les plus récentes produites par le Ministère de l'Environnement et de la Protection de la Nature du Sénégal, la réduction des forêts " incluant les ressources végétales et animales sauvages situées sur des terres non agricoles et non bâties " est de l'ordre de 45000 ha en moyenne par an pour la décennie 1990-2000 et de 40000 ha par an pour la décennie 2000- 2010 (MEPN-CEPS, 2011). Cette régression, somme toute importante mais qui ralentit, prend sa source dans la conjugaison de nombreux facteurs (sécheresse, feux de brousse, défrichements agricoles et approvisionnement en combustibles ligneux). Jusqu'en 1990, les zones de production de charbon incluaient Saint Louis, Louga et Kaolack en plus des régions de Kolda et Tambacounda situées respectivement à 693 et $461 \mathrm{~km}$ au Sud de la capitale Dakar. Jusqu'en 1997, elles intégraient encore Kaolack, dans de faibles proportions, mais elles ne comprennent plus que les deux dernières zones depuis la campagne de 1998. A partir de ce constat, conjugué aux nouvelles lois sur la décentralisation, les bailleurs de fonds ont invité l'Etat à réformer le système de production forestière en intégrant l'idée d'une gestion rationalisée basée sur l'aménagement des forêts dans un souci de durabilité. Dans le contexte de la multiplication des aménagements, la région de Ziguinchor, en basse Casamance, jusque là épargnée, est récemment venue s'ajouter aux deux autres régions de production.

\section{Le système de quota : bref rappel historique de la structuration de la filière du charbon de bois}

Depuis les indépendances, dans les pays francophones d'Afrique de l'Ouest et du Centre, les dispositifs de gestion des ressources naturelles étaient caractérisés par une centralisation, une gestion directe et répressive de la part des services forestiers nationaux, poursuivant la politique coloniale d'exclusion des usagers de l'exploitation de la ressource et

${ }^{6} \mathrm{Cf}$. Boutinot, 2002. Il faut prendre ces pourcentages avec précaution. Bien qu'ils émanent de la DEFCCS, à travers le PROGEDE, ils ne compteraient pas les zones de forêts claires et des reliques de forêts denses situées dans le sud du pays en Casamance, d'après Ribot, 1990 :145. 
limitant ces derniers à l'usufruit pour les usages domestiques (Bergeret, 1995 ; Buttoud, 1995 ; Bertrand, 1998 ; Ribot, 1990, 1999 et 2001). L'exploitation forestière proprement dite et l'histoire de la production du charbon de bois au Sénégal nous rappellent ainsi leur lien étroit avec la mise en valeur économique du pays à l'époque coloniale. Le premier charbonnier est apparu à la faveur de la construction du chemin de fer DakarBamako. La main-d'œuvre réquisitionnée à l'époque venait de la Guinée voisine : des Peuls du Fouta Jalon employés au Sénégal par des Peuls de caste noble (Ribot, 1999). Ensuite, la politique de l'Etat indépendant a commencé à réglementer l'exploitation à partir d'un décret de loi en 1965 (65-078 du 10 février 1965) instituant l'obtention d'un permis de coupe (art. D 38 et 39), d'un permis de circulation (D 42) et d'un permis de dépôt (D 43) pour toute activité commerciale d'exploitation des ressources ligneuses. La loi de 1974 a, de plus, obligé l'exploitant forestier à se munir d'une carte professionnelle (L 74-46 du 18 juillet 1974. Art. L 28). Puis, dès 1980, un système de quota a été explicitement institué par la Direction des Eaux et Forêts, de la Chasse et de la Conservation des Sols (DEFCCS) afin de réguler la production face au nombre grandissant des patrons charbonniers. L'Union Nationale des Coopératives d'Exploitants Forestiers du Sénégal (UNCEFS) a été créée en 1981 et, dès 1983, les patrons charbonniers ont du se regrouper en coopératives. Mais ces dernières sont venues s'ajouter, plus qu'elles ne se sont substituées, aux sociétés privées des premiers patrons charbonniers qui ont continué de produire individuellement (Ngom, 2006 :14) les plus gros volumes (Ribot, 1990 :159). En 1987, le Sénégal comptait 83 coopératives et 8 entreprises privées (République du Sénégal, 1987). Puis, le gouvernement a incité les exploitants à créer des groupements d'intérêt économique (GIE). En 2004, le nombre d'organismes d'exploitation de charbon de bois s'élevait à 146, se répartissant en $53 \mathrm{GIE}, 85$ coopératives et 8 entreprises privées (République du Sénégal, 2005). En quelques quinze ans, le nombre d'organismes a peu augmenté et les créations ont concerné essentiellement des GIE ${ }^{8}$. En 19931994, des soutiens financiers ont été accordés afin d'aider à démarrer les activités dans le cadre de ces nouvelles structures, sans grand succès et

8 Le nombre de patrons actifs a été évalué à 600 en 2006 par Ribot. Environ 4800 surghas 120 contremaîtres et intermédiaires informels, 300 coxeurs ou grossistes et 850 détaillants. 1 . est à noter qu'une coopérative type peut comprendre entre 30 et 100 patrons (Ribot, 1990 : 159). 
sans suite car " les moyens accordés ont été utilisés par les exploitants forestiers à des dépenses privées et non professionnelles » (Cilss, s.d : 29, Ngom, 2006 : 29). Le nombre d'organismes autorisés à l'exploitation a néanmoins grandi progressivement lors de la dernière décennie puisqu'ils étaient 191 autorisés à l'exploitation en 2009, 211 en 2010 et 249 en 2011 (J.O 2009, 2010, 2011). Ils atteindraient même le nombre de 284 organismes en 2013 (le-dakar.com). Le montant du quota national alloué à la production de charbon de bois a quelque peu suivi cette courbe, bien que de façon insuffisante selon l'UNCEFS'.

La différence entre les trois structures - coopérative, GIE et entreprise privée - tient au fait que les redevances ne s'engagent pas au même moment de la production. Les sociétés et les GIE doivent payer au moment de leur création une redevance de l'ordre de 100000 FCFA, quelque soit le nombre de surghas employés. En revanche, les coopératives paient une taxe de 500 Fcfa pour chaque membre, et affichent le nombre de leurs surghas. Les surghas paient une taxe de 3000 Fcfa auprès du service forestier pour obtenir une carte professionnelle que les agents peuvent contrôler sur les chantiers de carbonisation en forêt (Ngom, 2006 :16). La capacité de production d'une coopérative se trouve ainsi calculée en fonction du nombre de surghas déclarés par les membres. Sachant qu'un surgah est en mesure de produire 300 quintaux, il est donc indirectement l'indice de mesure de la capacité de production de la coopérative et, partant, du montant du quota autorisé. Cette forme d'organisme a, en fait, été créée afin de mieux contrôler et d'orienter la production mais sa faible adoption par les patrons charbonniers témoigne de la volonté de l'oligopole de résister à l'élimination du système du quota. De plus, chaque année, une part de quota, appelée «quota d'encouragement ${ }^{10}$, laisse une marge de manœuvre supplémentaire au service forestier dans ses modes d'attribution. Moyen de pression politique pour les uns, moyen de capter des rentes

9 Le quota national est passé de 500000 quintaux en 2005 à 741000 quintaux en 2009 ; 679314 q. en 2010 et 778228 q. en 2011. Cf. Les arrêtés ministériels MEPN-DEFCCS fixant les modalités d'organisation de la campagne d'exploitation forestières des années concernées.

10 ॥l existe des quotas d'encouragement qui sont distribués et qui ne sont pas systématiquement ni nominativement spécifiés dans les arrêtés annuels portant organisation de la campagne d'exploitation forestières. Mais quand ils le sont (tel que dans les arrêtés de 2000 et de 2002), ce sont toujours les 8 entreprises privées les plus importantes qui reviennent. 
pour les autres, le quota perdure et reste lié à un système de patronage et de mise au travail d'employés, les surghas; lesquels sont contraints dans des relations de " crédit-travail » avec leur patron (Bardhan, 1980, cité par Ribot, 1990 : 155). Il demeure le moyen d'accès à la ressource, délivré par le Ministère de l'environnement et de la protection de la nature (Ngom, 2006 : 14). Mais il se trouve désormais mis en question à travers les politiques d'aménagement forestier et les processus de transfert de gestion aux collectivités locales élues.

\section{La logique des politiques d'aménagement forestier}

La logique des aménagements forestiers s'appuie en partie sur des raisons économique et écologique. Elle consiste à structurer la filière de production selon les zones de prélèvement dont la ressource a été évaluée en fonction de son potentiel écologique de régénération. C'est à partir de 2004 que les premiers aménagements des espaces forestiers ont été réalisés par le PROGEDE dans les régions ouvertes à la production de charbon. Ils suivent une logique d'ensemble, à l'instar des expériences de la Banque Mondiale au Niger ${ }^{11}$ et au Mali ${ }^{12}$, selon laquelle les zones aménagées prennent leur sens par rapport aux zones non aménagées grâce à un système de taxation différentielle auquel sont soumis les exploitants. Une première taxe différentielle avait été instituée au Sénégal par un décret datant du 9 juillet 1996 qui fixait à 700 Fcfa par quintal (art. 23) au lieu de 1400 Fcfa la redevance aux exploitants qui orienteraient leur production de bois et de charbon de bois dans des zones bénéficiant d'un aménagement forestier. Mais ces mesures étaient restées au stade de l'intention du fait qu'il $n^{\prime}$ existait pas encore d'espaces véritablement aménagés ${ }^{13}$. Avant le décret de 1996 cependant, le montant des taxes était minime et les lieux de production n'étaient pas limités et peu contrôlés (Cf. tableau 1). La taxe ne structurait pas le système de production. Depuis 1996, la volonté des services forestiers est d'obliger progressivement les exploitants à produire

${ }^{11} \mathrm{Cf}$. Antona et al., op.cit.

${ }^{12} \mathrm{Cf}$. Mbodj, F., dans ce même numéro.

13 Les premières orientations spatiales des exploitants ont été expérimentées en 1979 dans la forêt de Koupentoum, (région de Tambacounda) mais sans réel inventaire écologique. Elles ont en fait donné lieu à des dégradations massives autour des villages (Ribot 1990 : 154). 
dans les zones qui font l'objet d'un aménagement durable. Le code forestier de 1998 le stipule explicitement dans son article 66 : "Afin de permettre la continuité dans l'approvisionnement en charbon de bois des villes du Sénégal, l'exploitation sous sa forme consistant à allouer des quantités de charbon de bois par exploitant ou par organismes d'exploitation, reste possible dans les forêts non aménagées relevant de la compétence des collectivités locales pour une période de trois ans à partir de l'entrée en vigueur du présent code ». Autant dire que depuis 2001 le système du quota est dans l'illégalité (Ba, 2006).

\begin{tabular}{|c|c|c|c|}
\hline $\begin{array}{c}\text { Zones de production de } \\
\text { charbon de bois }\end{array}$ & Avant 1996 & $\begin{array}{c}\text { Décret 96 - 572 } \\
\text { du 9 juillet 1996 }\end{array}$ & $\begin{array}{c}\text { Décret 2001- } \\
217 \text { du 13 mars } \\
2001\end{array}$ \\
\hline Produits de défriche & $500 \mathrm{Fcfa}$ & $3400 \mathrm{Fcfa}$ & $2400 \mathrm{~F} \mathrm{cfa}$ \\
\hline $\begin{array}{c}\text { Zone non aménagée } \\
\text { (ZNA) }\end{array}$ & $150 \mathrm{Fcfa}$ & $1400 \mathrm{Fcfa}$ & $1200 \mathrm{Fcfa}$ \\
\hline Zone aménagée (ZA) & & $700 \mathrm{Fcfa}$ & $700 \mathrm{Fcfa}$ \\
\hline
\end{tabular}

Extrait du recueil des textes sur les collectivités locales au Sénégal (J.O. n 5698, p.0301)

Tableau 1 : Evolution des taxes par quintal de charbon de bois entre 1996 et 2001.

En 2001 une réévaluation des taxes à la baisse est apparue dans un nouveau décret, discriminant toutefois encore les zones de production selon leur aménagement - bien que toujours inexistant dans la réalité. Le service forestier, nécessairement sensible aux activités de lobbying des grands patrons charbonniers " anticipe parfois sur les préoccupations des exploitants pour ne pas toujours être désavoué par les politiques", ainsi que nous le précise Ngom (2006 : 33). La mise en œuvre de cette orientation politique vers les aménagements forestiers s'est finalement concrétisée dans le cadre du PROGEDE qui a réalisé 9 plans d'aménagement entre 2004 et 2008, couvrant 381074 ha de forêt, soit une superficie d'environ $20 \%$ des forêts communautaires ${ }^{14}$ (PROGEDE, 2008, République du Sénégal, 2008). Ces espaces aménagés ne représentent pas tout l'espace

\footnotetext{
${ }^{14}$ Les forêts communautaires, d'après nos calculs à partir des données disponibles, représentent plus de la moitié (55\%) de l'espace forestier, au moins pour la région de Tambacounda (IREF Tambacounda, 2005, Gestion du domaine forestier. ch I, p 28). La proportion est sensiblement la même dans la région de Kolda.
} 
potentiel de production de charbon de bois mais ils introduisent déjà une brèche dans le monopole des exploitants charbonniers traditionnels puisqu'ils obligent ces derniers à négocier avec les communautés rurales et les comités villageois l'accès à la ressource et à participer financièrement à la gestion forestière locale, à l'aide d'un pourcentage du prix prélevé sur chaque sac de charbon produit.

\section{La gestion participative}

Une seconde logique qui sous-tend la politique de l'aménagement et qui s'inscrit dans les processus de décentralisation, est celle de "la gestion participative " qui veut que les populations riveraines des forêts soient encouragées à s'engager activement dans les programmes mis en œuvre. D'une part, elles sont invitées à se regrouper en comité villageois de gestion et de développement (CVGD), à protéger les forêts et à obtenir, en contrepartie, un pourcentage des revenus générés par les activités d'exploitation des produits ligneux. D'autre part, certains villageois volontaires sont formés à la carbonisation afin de devenir producteurs de charbon de bois et bénéficier des revenus directs de cette activité.

Le PROGEDE est un projet de développement qui a permis au service forestier de maintenir son autorité sur les forêts communautaires tout en répondant aux exigences de la promotion participative ${ }^{15}$ et en ouvrant l'activité aux villageois. Ainsi, le PROGEDE a organisé les populations en comités à l'échelle des villages riverains des massifs forestiers délimités pour les aménagements. Quelques 300 CVGD ont été constitués dans les forêts aménagées. Le choix de l'échelle villageoise renvoie à plusieurs ordres de justification. De manière patente, elle est considérée comme une base d'intervention à dimension humaine et, dans la vulgate du monde développementaliste, elle est l'expression d'une idéologie communautariste notamment lorsqu'il s'agit de gestion des ressources naturelles (Bako Arifari et Le Meur, 1998) et apparaît, de manière récurrente, comme

\footnotetext{
15 Depuis les textes de lois de la décentralisation et le nouveau code forestier, le service des Eaux et Forêts au Sénégal a engagé de nombreuses activités de formation pour tous ses agents aux approches participatives. Cf Boutinot, 2002. Sur l'analyse de ces approches voir aussi Boutinot et Diouf, 2006.
} 
le lieu idéal de la démocratie. Mais, en dehors de cet affichage idéologique, ce choix n'est pas sans lien avec le système de production de charbon de bois. Historiquement, avant la décentralisation et le transfert d'autorité aux Conseils Ruraux, la distribution des quotas suivait un cheminement qui sollicitait directement l'accord des chefs de village, à une échelle infralocale. Les agents de terrain des services forestiers avaient l'habitude de faire auprès d'eux "les salutations $d^{\prime}$ 'usage ${ }^{16}$ qui conviennent en pareille circonstance et qui sont "la moindre des choses" avant de venir installer les employés des grands patrons: "Depuis que je suis là, je n'ai pas rencontré de village qui ait refusé, car il y a un travail de rapprochement de la part de l'agent qui se fait avant l'installation des surghas" a ainsi déclaré un chef de brigade forestière ${ }^{17}$. L'ancienneté des relations entre les patrons charbonniers, les agents forestiers et les chefs de village peut expliquer ainsi que le choix de l'échelle villageoise reste étroitement lié aux stratégies d'accès à la ressource.

Cependant, la création des comités villageois de gestion et de développement (CVGD), fédérés dans un comité inter-villageois (CIVGD) dans le cadre du projet d'aménagement forestier, avait pour but de sensibiliser et d'organiser les populations villageoises dans des activités de surveillance et d'entretien des espaces forestiers selon les modalités prévues dans le plan d'aménagement. Cette démarche avait également comme objectif de les faire participer directement à la production du charbon de bois et de les inviter à bénéficier de ses retombées financières. Ainsi, les villages comme les communautés rurales sont intéressées aux retombées économiques que l'aménagement forestier leur procure, soit en captant une ristourne sur la production locale de charbon réalisée par les villageois résidents, soit en captant la ristourne sur la production effectuée par les surghas venus de l'extérieur pour le compte d'un patron charbonnier urbain.

Ces procédures font quelque peu entorse aux lois sur la décentralisation dans la mesure où les échelons des CVGD et du CIVGD n'existent pas en soi. Ils sont des comités ad hoc qui viennent capter une part des ristournes en lieu et place des Conseils Ruraux. C'est pourquoi les protocoles

\footnotetext{
${ }^{16}$ Entretien avec un chef d'une brigade forestière d'une région productrice de charbon de bois.

17 Idem.
} 
d'aménagement ont fait l'objet de négociations au cours de plusieurs réunions au village ${ }^{18}$ afin d'ajuster progressivement une clé de répartition des recettes potentielles respectant les différents groupes d'acteurs. Suite à de nombreuses négociations, parfois conflictuelles, au cours desquelles les présidents des communautés rurales concernées et les présidents de comités villageois ont fait valoir leurs droits, le code local de gestion de la forêt aménagée a finalement institué une clé de répartition des recettes, présentée dans le tableau 2 ci-dessous :

\begin{tabular}{|c|c|c|}
\hline $\begin{array}{c}\text { Clef de } \\
\text { répartition }\end{array}$ & Producteur local & Contractuel \\
\hline Producteur & $80 \%$ & $75 \%$ \\
\hline Conseil Rural & $10 \%$ & $10 \%$ \\
\hline CVIGD & $6 \%$ & $5 \%$ \\
\hline CVGD & $4 \%$ & $10 \%$ \\
\hline Total & $100 \%$ & $100 \%$ \\
\hline
\end{tabular}

Tableau 2: Clef de répartition des recettes d'exploitation forestière dans une forêt aménagée

Les producteurs locaux sont les villageois qui participent à l'entretien et la surveillance des forêts de leur communauté rurale en même temps que, nouvellement formés à la production de charbon de bois, ils sont devenus, pour certains d'entre eux, des producteurs. Les contractuels sont les patrons charbonniers qui continuent à installer leurs surghas dans la forêt aménagée, étant entendu que les villageois ne sont pas (encore) assez nombreux à produire. Une taxe de $25 \%$ du prix du sac de charbon est ainsi désormais prélevée sur la production des patrons charbonniers au bénéfice des institutions locales (CVGD, CIVGD et Conseil Rural). Elle vient s'ajouter à la redevance de $700 \mathrm{Fcfa} /$ quintal de charbon de bois que l'exploitant paie au service forestier à l'échelon national. II faut noter que cette clef de répartition locale des recettes peut changer d'une forêt aménagée à une autre, selon le rapport de force entre les producteurs

\footnotetext{
${ }^{18}$ Nous présentons, ici, le cas d'une des quatre forêts dont les plans d'aménagements ont été opérationnels en 2004.
} 
villageois, les présidents des Conseils Ruraux ${ }^{19}$, les CVGD et CIVGD, et les patrons charbonniers extérieurs.

\section{La « libéralisation » et l'intégration des villageois} dans la filière $d u$ charbon de bois

La gestion participative, sollicitée à travers la mise en aménagement des forêts communautaires, sous-entendait, à terme, la possibilité pour les villageois d'intégrer à leur propre compte la filière de charbon de bois, en lieu et place des surghas employés par les grands patrons. Le discours des animateurs du projet reprenait celui qui officiellement accusait ces derniers de détruire les forêts sous "l'œil impuissant " des populations riveraines qui ne jouissaient jusque là que de l'usufruit des produits forestiers, sans bénéficier des retombées financières. Afin de compenser les efforts déployés par les villageois dans l'aménagement des espaces forestiers, l'entretien des pare-feux, le reboisement, la surveillance et la lutte contre les feux de brousse, le prix des sacs de $50 \mathrm{~kg}$ de charbon produit par les premiers villageois formés à la carbonisation, avait été fixé par le PROGEDE à $1000 \mathrm{Fcfa}$, au lieu de 700 ou $750 \mathrm{Fcfa}$ et ceci, dès la première campagne de production en 2004. Les villageois restaient cependant soumis, comme par le passé, au monopole des patrons charbonniers qui seuls détiennent des cartes professionnelles officielles, des permis de circulation et de dépôt, tous documents légalement nécessaires pour commercialiser la production ${ }^{20}$.

En effet, le statut des CVGD n'autorise pas leurs membres à obtenir une licence d'exploitation. Il est d'ailleurs apparu, au cours des deux années qui ont suivi (2005-2007), que l'écoulement du charbon de bois produit par les villageois eux-mêmes posait de sérieux problèmes liés aux difficultés rencontrées par le PROGEDE pour trouver des acheteurs au prix rémunérateur proposé. Alors que le PROGEDE avait formé les villageois à la production et appuyé la commercialisation, il touchait là à ses limites. Les expériences déconcertantes de gâchis de certaines quantités produites - volées ou détériorées par les pluies durant l'hivernage de 2004 - n'ont pas satis-

\footnotetext{
${ }^{19}$ Il peut arriver qu'une forêt soit à cheval sur deux Collectivités rurales (à savoir Saré Bidjé et Ndorna) ainsi qu'il en était au départ pour la forêt de Saré Gardi en Haute Casamance.

${ }^{20}$ Le transport, intégré aux entreprises des exploitants les plus puissants, est encore un phénomène spécifique au système oligopolistique des patrons charbonniers sénégalais.
} 
fait les villageois. A ce propos, le rapport d'un animateur précise de manière éloquente : "Ce qui a ralenti le travail de ramassage [de bois mort] ${ }^{21}$, c'est le fait que les premières meules défournées depuis juin n'ont pas pu être écoulées. (...) C'est l'éternel problème de mévente du charbon. Actuellement, on ne sait plus que dire aux comités qui ont déjà leur charbon exposé. Des fois, on a des problèmes de s'y rendre même si on s'efforce de le faire tout en se bouchant les oreilles ${ }^{22}$. En réalité, l'écoulement d'une certaine partie de la production n'a été possible qu'avec l'intervention du Ministre de l'Environnement en mission dans la région en 2004, qui a sommé le président de l'UNCEFS de trouver des acheteurs pour les produits invendus du PROGEDE et, pour le remercier, lui a octroyé un quota supplémentaire de 400 permis de 150 quintaux chacun. Mais il s'agissait là d'une mesure exceptionnelle et isolée $e^{23}$.

Dans le rapport de la mission d'évaluation du PROGEDE réalisée par la Banque Mondiale, il est apparu de nouvelles conditionnalités pour assainir la situation des producteurs villageois du PROGEDE. Dans ce que les représentants de la Banque Mondiale appellent un souci de libéralisation économique de la filière, les CVGD ont été invités à se constituer en GIE de manière à obtenir une licence d'exploitation et à intégrer la filière jusqu'au commerce de détail en zone urbaine. Malgré la pression du bailleur de fond, force a été de constater que non seulement il n'est pas aisé de se constituer en GIE, mais ceux qui l'ont fait n'ont pas pu obtenir davantage de licence. En revanche, les villageois d'un massif aménagé voisin n'ont pas attendu d'être constitués en GIE pour aller vendre, à leurs propres frais ${ }^{24}$, leur production à Dakar. Ainsi que nous le commente un agent du PROGEDE, "ils ont goûté et c'était bon ". Ils ne voulaient plus vendre aux exploitants extérieurs. Fort de ce mouvement, il convenait d'attribuer des quotas de production spécifiques aux villageois désormais formés à la carbonisation et devenus producteurs directs. Dès lors, un quota de

${ }^{21}$ Avant de couper les arbres d'une parcelle, le bois mort est d'abord utilisé pour la carbonisation.

${ }^{22}$ Rapport mensuel de l'animateur d'une des forêts aménagée, Juillet 2002, PROGEDE.

${ }^{23}$ Cette mesure a été justifiée par la crainte d'une pénurie en période de fête de la Tabaski (Boutinot, 2004).

${ }^{24} \mathrm{D}$ 'après l'animateur du PROGEDE, les villageois ont vendu une partie de leur bétail pour financer le transport de leur production jusqu'à Dakar. 
160000 quintaux a été alloué aux zones aménagées du PROGEDE, sur les 500000 du quota national. Ainsi, ces villageois pionniers d'un des massifs aménagés ont-ils pu épuiser rapidement leur quota de 7500 quintaux en vente locale et 19500 quintaux en vente aux centres urbains en 2005 (Ngom, 2006: 18) où le prix du sac de $50 \mathrm{~kg}$ variait entre 3500 et 4500 Fcfa.

Les Zones de Production Contrôlée, entre le hasard écologique et la nécessité politique

Les ZPC apparaissent tardivement au Sénégal et seulement après la mise en œuvre des premiers plans d'aménagement du PROGEDE. Elles trouvent difficilement leur justification au sein même de la logique d'aménagement et de ses taxations différentielles. Conséquemment, il n'est pas aisé pour les services forestiers de justifier leur mise en œuvre à partir d'un argumentaire écologique. Les ZPC révèlent, si besoin était, l'enjeu économique et politique de la gestion de la filière et les dissensions au sein des services forestiers.

Les ZPC et les aménagements simplifiés pour contrôler l'accès aux ressources

Comme nous l'avons évoqué en introduction, les zones de production contrôlées (ZPC) se constituent dans les espaces forestiers des communautés rurales, en dehors des forêts aménagées par le PROGEDE. Et, bien que ces ZPC fassent l'objet d'un aménagement simplifié, elles restent malgré cela dans la même catégorie fiscale que les zones aménagées. On est en droit de penser que cette catégorie de zone de production nouvelle cristallise, en même temps qu'elle illustre, les tensions dans un service forestier "à deux vitesses » (Olivier de Sardan, $1998: 168$ ) divisé entre les projets qui bénéficient des financements des bailleurs de fonds et le service traditionnel qui reste bien mal loti. Suite aux effets des aménagements menaçant les intérêts des exploitants forestiers et considérant le poids politique et financier de ces derniers, les ZPC sont advenues comme une formule alternative, voire un antidote aux dispositifs mis en place par le PROGEDE.

Par ailleurs, si les procédures de contractualisation dans les ZPC semblent les mêmes que dans les zones du PROGEDE, elles présentent quelques nuances. Tout d'abord les populations des villages en bordure 
des forêts ne sont pas constituées en comité de gestion (CVGD) pour négocier les contrats et les clés de répartition avec les exploitants urbains. D'autre part, la production du charbon dans ces forêts est exclusivement réservée à ces exploitants ; les villageois participent plutôt aux activités d'entretien moyennant $200 \mathrm{Fcfa}$ qui sont théoriquement versés pour chaque sac de $50 \mathrm{~kg}$ produit et qui sont destinés aux conseils ruraux. Et si les institutions locales (CVGD, CIVGD) créées dans le dispositif des Zones Aménagées par le PROGEDE et le conseil rural retiennent $25 \%$ du prix du sac vendu aux exploitants pour le financement du développement local, en revanche dans les ZPC, c'est un prélèvement fixe de $200 \mathrm{Fcfa}$ par sac qui revient à la communauté rurale et le fonds ainsi créé est, de surcroît, géré conjointement par les services traditionnels des Eaux et Forêts et l'Union des Exploitants forestiers ${ }^{25}$.

Dans un document datant d'avril 2005, I'Inspection Régionale des Eaux et Forêts de Tambacounda précise que c'est dans le contexte de la mise en place des plans d'aménagement participatif du PROGEDE que les ZPC sont constituées : "le service des Eaux et Forêts a pris l'initiative de rationaliser la production de charbon, en plus des actions menées par le PROGEDE dans les différentes forêts communautaires en association avec les collectivités locales concernées, par la mise en place de Zones de production contrôlées en partenariat avec les exploitants traditionnels " (IREF, $2005: 3$ ). Comme I'indique leur nom, elles sont, en définitive, justifiées pour " assainir » la production et mieux la contrôler ${ }^{26}$. Un responsable de la DEFCCS nous précisait ainsi que, comme dans les zones aménagées, il y a, dans les ZPC, un aspect " sécurisation » de l'exploitation. Ainsi "le surgah ne peut pas disparaître avec le produit, l'argent etc... Il est contraint et contrôlé par parcelle, par zone. Et il y a une "traçabilité " du produit qui sort des zones aménagées et des ZPC. L'origine du produit est marqué au stylo sur le titre d'exploitation ». Une telle sécurisation ne peut que rassurer.

${ }^{25}$ Pour la ZPC de Missirah (région de Tambacounda) l'argent du Fonds est géré par le chef de brigade des E\&F (USAID, 2005, p. 5).

26 « Plan de gestion de la ZPC de Missirah » op.cit. p 14. 


\section{Les ZPC détachées de l'argumentaire écologique?}

Mais ce sont également la durée, la cherté et la complexité des aménagements réalisés qui ne permettent pas, selon certains agents rencontrés, d'envisager la multiplication des procédures du PROGEDE sur tout le territoire. "S'il y a des ZPC, c'est parce que les moyens manquent, sinon tout serait en zone aménagée et les villageois produiront " nous disait un responsable régional des Eaux et Forêts il y a quelques années ${ }^{27}$. Ainsi, même du côté des services forestiers qui les mettent en œuvre, ces zones ne sont pas considérées comme aménagées. Créées de manière diligentée et considérées en marge de la logique d'aménagement, les ZPC ont pu faire l'objet de définitions pour le moins variées notamment du point de vue des agents qui ont œuvré dans le dispositif du PROGEDE. Elles leur paraissent incongrues, ce que nous révèlent les acronymes qu'ils utilisent pour les qualifier : tantôt ZPTA, littéralement "Zones Pas Trop Aménagées ", ou bien ZPTT, "Zones Pas Très Traditionnelles ». Mais, ainsi que nous le présentait un agent du secteur: "la direction des Eaux et Forêts nous demande de faire des ZPC, alors nous y allons". Au nombre de 4 en $2005^{28}$, ces ZPC sont amenées à s'étendre puisqu'elles entrent désormais dans les performances environnementales fixées par la DEFCCS dans les critères et indicateurs de gestion qui forment les conditionnalités de l'appui budgétaire mis en place par les bailleurs de fonds ${ }^{29}$. Cet appui budgétaire venait contredire les approches par projets, telle celle de la Banque Mondiale, dont la mise en œuvre est considérée par certains un peu en marge des politiques publiques. Le Ministère de l'Environnement sénégalais entendait ainsi étendre le principe d'aménagement simplifié à toutes les forêts communautaires productrices de charbon de bois, en dehors des formes complexes du PROGEDE, dont le coût à l'hectare a été évalué à 4722 Fcfa (Djigo, 2003: 51). Le coût de l'aménagement à l'hectare en ZPC peut être évalué, d'après les documents de l'inspection forestière (Linde et al, 2005 : 47), à 1258,48 Fcfa. Ce dernier est ainsi trois fois inférieur à celui

\footnotetext{
27 Entretien avec un agent des E\&F.

28 Dont 2 dans la région de Kolda et 2 dans la région de Tambacounda (bien qu'une seule ait réellement fait l'objet d'un protocole de gestion). Elles couvrent des superficies de 2700 ha et de 3000 ha dans la région de Kolda et de 1904 ha dans la région de Tambacounda.

${ }^{29}$ En ce qui concerne la gestion de l'Environnement, l'appui budgétaire est mis en œuvre par les Pays-Bas au Sénégal. Il est à noter que l'ambassade des Pays-Bas s'est retiré du PROGEDE en 2005.
} 
d'une zone aménagée du PROGEDE. Mais, afin de justifier la taxation préférentielle dont bénéficient les ZPC au même titre que les zones aménagées, nombre de cadres des services forestiers soutiennent que les ZPC font l'objet d'un plan d'aménagement aussi rigoureux et respectueux des normes environnementales que celles établies dans le PROGEDE. Et seul le fait que les aménagements du PROGEDE soient intégrés (c'est-à-dire qu'ils incluent des " séries » ou des subdivisions de l'espace pour réglementer les autres activités de production : agriculture et élevage) les distinguerait des ZPC qui, elles, sont focalisées sur la seule production de charbon de bois.

Pourtant, même si des critères minimaux sont stipulés dans les textes instituant les ZPC (utilisation de la meule casamançaise comme technique de carbonisation améliorée, diamètre des arbres à respecter, $50 \%$ des essences forestières coupées), des points notables les éloignent de la rigueur d'un plan d'aménagement. D'une part, les inventaires forestiers des ZPC, quand ils ont été réalisés, ont été calculés par extrapolation des données construites par le PROGEDE à d'autres sites forestiers. Or, si ces derniers sont sous la même latitude et sont composés approximativement des mêmes essences, ils ne présentent pas le même passé de production. Les ZPC sont créées sur les terres de jachères ou les espaces de brousse communautaires, faisant fi des multi-usages mais aussi de la durabilité de la ressource.

D'autre part, les assiettes de coupe ont été délimitées par les services forestiers sans toujours faire l'objet d'information préalable et précise ou bien, quand elles l'ont été dans le cadre obligatoire des délibérations du Conseil Rural, c'était au moyen de procédures rapides ${ }^{30}$, non sans une certaine pression. Mais la démarche officielle veut que ce soit les communautés rurales qui sollicitent les services forestiers pour établir un plan d'aménagement. Ce qui fait dire au Chef de la division aménagement et exploitation forestière de la DEFCCS qu'on ne peut guère penser qu'elles soient opposées à l'exploitation ${ }^{31}$. De son côté, le représentant de I'UNCEFS rappelle qu'avec la décentralisation, la responsabilisation des

\footnotetext{
${ }^{30}$ Cf. Procès verbal N ${ }^{\circ} 04$ du Conseil rural de Missirah lors de sa séance du 29 /06/ 2006. 20 élus présents sur 32. Accord pour la ZPC à 13 voix sur 20.

${ }^{31}$ Cf. le film Ribot Debates Forester and Merchant on Senegal Television, 2011, publié sur youtube le 8 août 2013.
} 
populations et l'assiette de coupe établie par les services forestiers, il n'y a plus besoin de quota ni d'autorisation préalable des conseils ruraux ${ }^{32}$. On est de fait en droit de se demander si les ZPC ne seraient pas susceptibles d'ouvrir la voie à des formes futures de mise en concessions privées des ressources forestières au Sénégal, tel que le préconise le document du programme régional de promotion des énergies domestiques et alternatives au Sahel (Ngom, 2006).

Enfin, le contrôle technique de l'exploitation dans les ZPC relève du service traditionnel des forestiers qui dispose de peu de moyens humains et matériels. Chaque zone de coupe étant attribuée à un organisme exploitant, le martelage des arbres à couper (signalement par une marque à la peinture sur le tronc) est donc fait par ceux-là mêmes qui doivent produire et c'est à la charge de ces derniers qu'incombent, en définitive, la surveillance et le contrôle des critères environnementaux de la production. Le président de I'UNCEFS précisait d'ailleurs que les patrons ont eux-mêmes formés les gens sur place ${ }^{33}$. Ainsi, selon un agent technique, avec des outils de mesure ad hoc ${ }^{34}$ confectionnés pour éviter de faire le calcul des diamètres des arbres à signaliser, "la tricherie est possible " ${ }^{35}$. Dans les ZPC, les populations riveraines des forêts ne participent pas à la production; elles se voient reléguées à nouveau au rang d'usufruitiers, voire de maind'œuvre d'entretien ou, simplement, de faire-valoir.

Ainsi, la différence de procédure entre les deux types de zone est sensible. De plus, une contradiction se donne à lire entre les justifications selon lesquelles les ZPC sont créées pour remplacer les pratiques compliquées, longues et surtout trop coûteuses des aménagements du PROGEDE et le fait de justifier a posteriori ces ZPC sur le respect des critères d'aménagement les plus complexes.

\footnotetext{
${ }^{32}$ Idem.

33 Ibidem.

34 II s'agit d'une fourche confectionnée avec des branches dont l'écart doit correspondre au diamètre de l'arbre autorisé à la coupe.

35 Notons que les formations à la coupe du bois et aux techniques de carbonisation améliorées dispensées dans le cadre du PROGEDE ne ciblaient que les villageois résidents et non les "surgahs " des exploitants afin de ne pas inciter ces derniers à développer leurs activités (CILSS, sd. : 29)
} 


\section{Conclusion}

De fait, les ZPC sont apparues comme des instruments de gestion dont les justifications naviguaient entre le hasard écologique et la nécessité politique. Mais elles furent surtout un moyen politique de compromis entre les exigences de la décentralisation, le nombre grandissant de producteurs des comités villageois et le monopole des grands patrons charbonniers. L'augmentation du nombre des organismes producteurs de charbon de bois est, en effet, liée à l'entrée des comités villageois sur le marché (Cissé, 2013). C'est bien à partir de 2005 que les populations locales organisées en comités ont été autorisées à produire du charbon de bois. L'arrêté ministériel de 2006 fixant les modalités d'organisation de la campagne d'exploitation forestière ( $N^{\circ} 2344$ MEPN-DEFCCS en date du 13 avril 2006, art. 7) précisait même qu'en zone non aménagée, les organismes d'exploitants sont installés par le service forestier et qu'en zone aménagée, l'exploitation est réalisée par les populations organisées en structures de gestion, et également par les organismes contractants, c'est-à-dire ceux qui établissent un contrat avec les communautés rurales. Cette précision qui distribue les producteurs et exploitants en fonction des zones disparaitra toutefois dans les arrêtés suivants. Si, depuis cette date, les arrêtés ministériels distinguent les forêts aménagées des forêts non aménagées, il n'est pas fait mention de ZPC. L'évolution des rapports de force a fait apparaître depuis la fin de la première phase du PROGEDE et la période de transition, des rapprochements sensibles au point où l'on ne distingue plus guère les zones de production. Et les zones aménagées comprennent désormais celles que certains appelaient les ZPTA ou ZPTT. Les frontières se sont brouillées entre les zones d'exploitation et rendent caduque la logique de la taxation différentielle.

"Le quota est mort, alors vive le quota "... II ne reste qu'à lui trouver un autre nom ${ }^{36}$, nous disait à l'époque et non sans humour, le directeur des Eaux et Forêts. L'autre nom est celui de contrat. Avec un tel glissement sémantique, les services forestiers tentent de maintenir un statu quo dans

\footnotetext{
${ }^{36}$ Selon les termes d'un cadre des Eaux et Forêts. Atelier National sur la gestion décentralisée des ressources forestières au Sénégal organisé par le programme WRI- CIRAD- CODESRIA le 8 mai 2006 à Dakar.
} 
le système de production car " il n'est pas intéressant de batailler à visage découvert ${ }^{37}$. Le contrat déplace le problème de l'aménagement du souci écologique vers la nécessité de redistribuer les quotas de production de charbon de bois entre un plus grand nombre de producteurs en accordant laborieusement une place à ceux qui n'avaient jusque là pas accès au marché. Mais le contrat est aussi ce qui permet aux patrons charbonniers de se " mettre en ordre " devant la loi forestière en négociant, avec les collectivités locales, l'accès à des zones de production. L'argument des patrons charbonniers est de dire, par la voix du président de I'UNCEFS, que la décentralisation ne doit pas être une contrainte et que tous les sénégalais, en tant que citoyens égaux, $\mathrm{n}$ 'ont pas besoin d'être résidents d'une communauté rurale pour y accéder ${ }^{38}$.

Depuis la campagne d'exploitation forestière de 2010, les populations locales produisent dans le cadre de structures locales de gestion des forêts aménagées (sous le sigle de SLGF, bien qu'il soit amputé du A) qui sont délégataires du pouvoir des présidents des communautés rurales concernées. Ces structures négocient avec l'UNCEFS et établissent dans un cahier des charges la part réservée aux producteurs villageois et celle attribuée aux exploitants. Comme il se doit, cependant, ce protocole reste visé par I'Inspecteur régional des Eaux et Forêts et le président du conseil rural, et le directeur national des Eaux et Forêt demeure l'arbitre en dernière instance. En 2011, I'UNCEFS a réussi à obtenir la réouverture des régions de Kaolack, Kaffrine et Fatick, plus proches de la capitale, fermées depuis 1998 à la production ${ }^{39}$. Tout change et rien ne change. Un détail pourtant : les populations locales productrices de charbon organisées en comités villageois et relevant d'une SLGF peuvent désormais obtenir une carte de producteur gratuite. Ce n'est pas encore la carte professionnelle digne de ce nom qui distingue encore les grands exploitants, mais cela permet aux comités villageois d'acheminer leur production jusqu'à la ville en toute légalité. Parfois même, d'anciens surghas désireux d'améliorer leur situation s'installent dans un village pour produire au sein d'un comité. Cela représente un double mouvement de concurrence pour les exploitants

\footnotetext{
${ }^{37}$ D'après un cadre de la DEFCCS à Dakar.

38 Cf. le film Ribot Debates Forester and Merchant on Senegal Television, 2001, publié sur youtube le 8 août 2013, op.cit.

${ }^{39}$ Arrêté ministériel N 1246 MEPN-DEFCCS en date du 3 février 2011, art. 20.
} 
traditionnels. L'argument avancé par ces derniers pour justifier le développement des ZPC avait trait à une supposée incapacité des comités villageois issus du PROGEDE à produire suffisamment de charbon pour alimenter les villes du Sénégal ${ }^{40}$. Les comités villageois qui produisent de plus en plus réduisent la portée de cet argument. Pourtant, la menace de pénuries de charbon dans les villes, et notamment dans la capitale, est toujours l'épée de Damoclès brandie par l'UNCEFS jusqu'à aujourd'huii ${ }^{41}$.

Le poids politique réel des grands patrons charbonniers et la difficulté ou l'appréhension des décideurs politiques et des services forestiers à assainir un tel système de production seraient-ils surestimés ? Et la capacité des comités villageois à pousser les verrous pour intégrer la filière sousestimée ? Les récentes réformes fiscales du nouveau gouvernement de 2012 et la question, en particulier, de l'augmentation des taxes sur la production de charbon de bois ont invité les grands exploitants à lancer un mot $d^{\prime}$ ordre de grève ${ }^{42}$. Mais ce mouvement $n^{\prime}$ a pas été totalement suivi et son caractère unitaire quelque peu contrarié. Les rapports de force seraient-ils en train de vaciller?

\section{Bibliographie}

BA, El H., 2006, Le quota est mort, vive le quota! Monograph series, Dakar, Codesria.

BAKO-ARIFARI, N. et LE MEUR, P.-Y., 2003, « La chefferie au Bénin : une résurgence ambiguë ", In : Perrot, C.H. et Fauvelle Aymar, F.X, (dir.), Le retour des Rois. Les autorités traditionnelles et l'Etat en Afrique contemporaine, Paris, Karthala : 125-143.

BARDHAN, O., 1980, "Interlocking factor Markets and Agrarian Development : A Review of issues ", Oxford Economic Papers, 32 (1) : 82-98.

\footnotetext{
${ }^{40}$ Entretien avec un fonctionnaire de l'IREF de Tambacounda, 2005.

41 "Personne ne peut prendre le risque d'une pénurie » rappelle $M$. Sow, président de I'UNCEFS en 2011, (Ribot Debates Forester and Merchant on Senegal Television, op.cit.)

42 Cisse, Serigne Mansour Sy, "Augmentation de la fiscalité sur le charbon : de exploitants forestiers maintiennent leur mot d'ordre de grève ", Le Soleil on line posté le vendredi 26 juillet 2013, www.lesoleil.sn.
} 
BERGERET, A., 1995, "Les forestiers coloniaux : une doctrine et des politiques qui n'ont cessé de 'rejeter de souche' ». In : Chatelin, Y \& Bonneuil C. (ed.), Nature et environnement Les sciences hors occident au XX e siècle, vol 3, Paris ORSTOM : 59-74.

BERTRAND, A., 1998, "Les marchés ruraux du bois énergie au Niger ", In : LavigneDelville, P., Quelles politiques foncières pour l'Afrique rurale ? Réconcilier pratiques, légitimité et légalité, Paris, Karthala - Coopération française : 526- 533.

BOUTINOT, L., 2002, «De la complexité de la décentralisation. Exemple de la gestion des ressources forestières au Sénégal ", Bulletin de l'APAD, 23.

BOUTINOT, L, 2004, Etude de la contribution du Progede à la gestion décentralisée des ressources naturelles, Contrat Progede- Cirad 001-04. Rapport final, décembre 2004, Dakar, Direction des Eaux et Forêts, 151 p.

BOUTINOT, L., et DIOUF, C.N., 2006, « Quand certaines approches participatives engendrent des formes ambiguës de mobilisation de la société civile. Quelques exemples à propos de la gestion des ressources naturelles au Sénégal ", In : Bertrand, A., Karsenty, A.et Montagne, P. (eds), L'Etat et la gestion locale durable des forêts en Afrique francophone et à Madagascar, Paris, Cirad - L'Harmattan : 195- 212.

BOURDIEU, P, 2002, [1984], Questions de sociologie, Paris, Editions de Minuit, 288 p.

BUTTOUD, G., 1995, La forêt et l'État en Afrique sèche et à Madagascar. Changer de politiques forestières, Paris, Karthala.

CISSE, S. M. S., 2013, « Augmentation de la fiscalité sur le charbon : de exploitants forestiers maintiennent leur mot d'ordre de grève ", Le Soleil on line posté le vendredi 26 juillet 2013, www.lesoleil.sn.

DJIGO, A.S., 2003, Etude de la fiscalité forestière au Sénégal, Dakar, Ministère de I'Environnement et de l'Assainissement - DEFCCS.

DURAN, P., 2004, "Génèse de l'analyse des politiques publiques ", In : Boussaguet $\mathrm{L}$, Jacquot $\mathrm{S}$ et Ravinet P. (Dir.), Dictionnaire de l'analyse de politiques publiques, Paris, Presses de Sciences Po, p 232- 242.

MINVIELLE, JP., 1999, La question énergétique au Sahel, Paris, Karthala, 171 p.

IREF de Tambacounda, 2005, Plan de gestion de la zone de production contrôlée de Missirah, document 1, avril, MEPN- DEFCCS, 31 p. 
NGOM, A., 1996, Quelques éléments de stratégie pour une exploitation rationnelle du charbon de bois, Observatoire des combustibles domestiques, Ministère de l'Energie, des Mines et de l'industrie, MEPN, Dakar.

NGOM A., 2006, Les professionnels du bois énergie au Sénégal. Programme régional de promotion des énergies domestiques et alternatives au Sahel (PREDAS), CILSS, Dakar, $38 \mathrm{p}$.

OLIVIER DE SARDAN, J.P., 1998, "Quelques réflexions autour de la décentralisation comme objet de recherche ", Bulletin de l'APAD, $16: 165-171$.

OLIVIER DE SARDAN, J-P., et Tidjani Alou, M., 2009, "Le local comme enjeu politique et enjeu scientifique, Introduction » In : Olivier de Sardan J.P. et Tidjani Alou M. (éds) Les pouvoirs locaux au Niger, Tome 1 : A la veille de la décentralisation, CODESRIA et KARTHALA, 2009, 379 p.

PROGEDE, 2008, Bilan des réalisations du PROGEDE, janvier 1998-décembre 2008, Dakar, Unité de coordination du programme, Ministère de l'environnement et de la protection de la nature, des bassins de rétention et des lacs artificiels, Ministère de l'énergie, $35 \mathrm{p}$.

RIBOT, J.C., 1990, " Politique forestière et filière du charbon de bois ", In : Bergeret, A., L'arbre nourricier en pays sahélien, Paris, Editions de la Maison des Sciences de l'Homme : 139-183.

RIBOT, J.C., 1995, Le contrôle local des forêts au Sénégal: vers des politiques participatives. Etude régionale (Afrique), Washington D.C., RPTES Banque Mondiale, $67 \mathrm{p}$.

RIBOT, J.C., 1999, Integral Local Development : Authority, accountability and entrustment, Natural Resource Management, Série de Documents de travail, Washington D.C., Banque Mondiale - RPTES, 73 p.

RIBOT, J. C., 2001, Historique de la gestion forestière en Afrique de l'Ouest: ou comment la science exclut les paysans, Londres, IIED, 17 p.

RIBOT, J., 2011, Ribot Debates Forester and Merchant on Senegal Television, Film co-directed by Pape Faye and Jesse Ribot, based on a script of Ribot, Dakar, mars 2011. https://www.youtube.com/watch?v=zp9t91rwBCo.

\section{Publications nationales officielles}

ANSD, 2011, Situation économique et sociale 2011, Agence Nationale de la Statistique et de la Démographie, Dakar, Sénégal, www.asnd.sn 
CILSS, (s.d ;), Les exploitants forestiers au Sahel, Synthèse des études nationales (2006 - 2007), Programme Régional de Promotion des Energies Domestiques et Alternatives au Sahel (Predas), Ouagadougou.

Journal officiel du Sénégal : Arrêtés ministériels fixant les modalités d'organisation de la campagne d'exploitation forestière :

LINDE (van der )M., DEME M., KESSLER J. J., LETTE H., LO M., WADE P.W. , 2005, Evaluation de l'appui budgétaire ciblé pour le secteur de l'environnement au Sénégal fourni par les Pays-Bas, Dakar, Ambassade des Pays-Bas, version finale 24/06/05.

$\mathrm{N}^{\circ} 2344$ MEPN-DEFCCS en date du 13 avril 2006. J.O N6297 du 16 septembre 2006.

$\mathrm{N}^{\circ} 40$ MEPNBLA-DEFCCS en date du 9 janvier 2009. J.O N6498 du 14 novembre 2009.

N³276 MEPNBLA-DEFCCS en date du 8 avril 2010. J.O N 6539 du 7 août 2010.

N 1246 MEPN-DEFC en date du 3 février 2011. J.O Nº582 du 16 avril 2011.

Ministère de l'Environnement et de la Protection de la Nature, Cellule d'études, de planification et de suivi, 2011, Cadre des dépenses sectorielles à moyen terme 2011-2013, Dakar. www.ceps.gouv.sn

République du Sénégal, 1987, Arrêté portant organisation de la compagne d'exploitation forestière pour l'année 1986-1987, nº00054/ MPN/ DEF/ DPF, Dakar.

République du Sénégal, 2005, Campagne d'exploitation forestières 2004. Rapport Bilan, MEPN/ DEFCCS Dakar, $19 \mathrm{p}$.

République du Sénégal, 2008, Impacts environnementaux et socio-économiques du programme de gestion durable et participative des énergies traditionnelles et de substitution (PROGEDE), Dakar, ISRA / CIS / CILSS.

USAID, 2006, Programme Agriculture-Gestion des ressources naturelles «Wula Nafa", Rapport sur la tenue du Forum communautaire sur la ZPC de Missirah, 5 mars 2006.

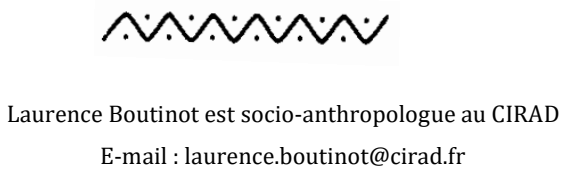


